\title{
PREPARAÇÃO E CARACTERIZAÇÃO DE RESÍDUO PÓS-INDUSTRIAL DE MADEIRA PLÁSTICA REFORÇADOS COM RESÍDUOS DE PÓ DE MADEIRA
}

\author{
W. F. P. DE PAULA ${ }^{1 *}$; L. P. DA SILVA ${ }^{1}$ \\ 1 Centro Universitário Estadual da Zona Oeste, 23097-220, Rio de Janeiro-RJ, Brasil \\ *wallacefernando@hotmail.com
}

Artigo submetido em 17/02/2016 e aceito em 28/04/2016

\begin{abstract}
RESUMO
O objetivo deste estudo é avaliar a viabilidade da produção de compósitos com resíduos de madeira plástica e pó de madeira com melhoria de propriedades. Resíduos pós-industriais a base de Polietileno de Alta Densidade (PEAD) utilizados como madeira plástica, denominados de resíduos de madeira plástica (RMP), foram beneficiados $\mathrm{e}$ misturados à resíduos de pó de madeira (RPM). As misturas foram preparadas com diferentes percentuais (em massa) de RPM (0, 10, 20, 30 e 40\%) em extrusora dupla-rosca modelo TeckTrill DCT 40, com L/D: 40 e com dez zonas de temperatura (mais a zona do cabeçote), compreendidas entre 135 e $220 \mathrm{oC}$, com velocidade de processamento de $60 \mathrm{rpm}$. Os perfis obtidos na extrusora foram analisados por torque,
\end{abstract}

densidade, dureza, índice de fluidez e Microscopia Eletrônica de Varredura (MEV). Os resultados de torque, dureza e densidade mostraram aumento proporcional com o percentual de RPM para todas as composições, sugerindo que a carga de RPM esteja atuando como reforço e preenchimento na matriz de PEAD. Quanto ao índice de fluidez, os compósitos de PEAD virgem/pó de madeira apresentaram redução com o aumento no teor de RPM, promovendo o aumento de viscosidade dos sistemas. Esta tendência não foi observada para os compósitos de RMP/RPM, devido provavelmente a presença de aditivos no RMP que mantém constante as viscosidades do sistema. As análises de MEV mostraram materiais homogêneos.

PALAVRAS-CHAVE: madeira plástica, resíduo de madeira plástica, resíduo de pó de madeira.

\section{PREPARATION AND CHARACTERIZATION OF WASTE WOOD POST- INDUSTRIAL PLASTIC REINFORCED WITH WOOD POWDER WASTE}

\begin{abstract}
The aim of this study is to assess the feasibility of production of composite plastic with wood waste and wood powder with improved properties.Post-industrial waste to be used as base High Density Polyethylene (HDPE) as used plastic wood, called plastic wood waste (RMP) were benefited and mixed with wood dust residues (RPM). The mixtures were prepared with different percentages (by mass) RPM $(0,10,20,30$ and $40 \%)$ in the twin-screw extruder model TeckTrill DCT 40, with L/D: 40 and ten temperature zones (more the head area), between $135^{\circ} \mathrm{C}$ and $220^{\circ} \mathrm{C}$, with a processing speed of $60 \mathrm{rpm}$. The profiles obtained in the extruder
\end{abstract}

were analyzed by torque, density, hardness, flow index and Scanning Electron Microscopy (SEM). The results torque, hardness and density showed a proportional increase with the RPM percentage for all compositions suggesting that the RPM load is acting as reinforcement and filling the HDPE matrix. As the melt index composite virgin HDPE/wood flour decreased with increasing RPM content, promoting the systems viscosity increase. This trend was observed for the composite RMP/RPM, probably due to the presence of additives RMP that keeps constant the viscosity of the system. The SEM analysis showed homogeneous materials.

KEYWORDS: plastic wood; plastic wood residues; wood dust residues. 


\section{INTRODUÇÃO}

Os polímeros são considerados os grandes vilões ambientais, pois podem demorar séculos para se degradar e ocupam grande parte do volume dos aterros sanitários, interferindo de forma negativa nos processos de compostagem e de estabilização biológica. Além disto, os resíduos poliméricos quando descartados em lugares inadequados, como lixões, rios, encostas, etc., causam um impacto ainda maior ao meio ambiente.

Vários aspectos motivam a reciclagem dos resíduos poliméricos contidos nos resíduos sólidos urbanos, a economia de energia, a preservação de fontes esgotáveis de matéria-prima, a redução de custos com disposição final do resíduo, a economia com a recuperação de áreas impactadas pelo mau acondicionamento dos resíduos, o aumento da vida útil dos aterros sanitários, a redução de gastos com a limpeza e a saúde pública e a geração de emprego e renda.

O objetivo deste estudo é avaliar a viabilidade da produção de compósitos com resíduos de madeira plástica e pó de madeira com melhoria de propriedades. Neste contexto, uma alternativa inteligente e ecologicamente correta para a problemática do descarte inadequado é a reutilização de resíduos pós-industriais a base de PEAD e resíduos de pó de madeira na formulação de compósitos com propriedades importantes.

Através da parceria da Universidade Estadual da Zona Oeste (UEZO) com a Companhia Municipal de Limpeza Urbana (COMLURB) foi possível viabilizar o projeto de pesquisa usando materiais $100 \%$ reciclados, sem aditivos, visando confirmar e eficiência de compósitos de madeira plástica com propriedades satisfatórias e ecologicamente corretas.

\section{REVISÃO DA LITERATURA}

\subsection{Madeira plástica}

A tecnologia de compósitos de madeira com polímero é uma prática antiga e com atuais avanços expressivos em mercado e tecnologia. Mundialmente já são produzidos industrialmente em diversas partes do mundo, no Brasil ainda demanda confiança no mercado. A cadeia produtiva de madeira e móveis gera significativa quantidade de resíduos que, embora muitas vezes seja aproveitada para alguns fins específicos, pode se constituir em problema de gestão ambiental, considerando também a alta inflamabilidade desse rejeito.

Correa (2003) aponta que estudos mercadológicos realizados nos EUA e na Europa sobre o uso de fibras celulósicas ou farinha de madeira como carga e reforço em termoplásticos revelam que a substituição da madeira convencional por compósitos termoplásticos apresenta-se como alternativa viável ao reaproveitamento de resíduos, com inúmeras vantagens:

Maior resistência à umidade e deterioração ambiental;

Resistência a pragas e insetos;

Podem ser extrusados em perfis com formatos diversificados;

Apresentam melhor estabilidade dimensional;

Resistência ao empenamento e trincas;

Possuem menor custo de manutenção de rotina;

Maior durabilidade em ambientes agressivos como marinas e piscinas; 
São totalmente recicláveis e imitam em aspecto a madeira;

Dispensam o uso de proteção superficial como tintas e vernizes.

\subsection{Resíduos de madeira}

O desperdício dos resíduos dentro das serrarias, não só representa um problema econômico, como também um grave problema ambiental. Estes podem se transformar em matéria-prima com diversas possibilidades de uso, através de técnicas simples e de baixo custo, reduzindo o volume de resíduos situados nas serrarias e, portanto aumentando sua receita (OLANDOSKI, 2001).

Em razão da variedade de espécies de madeira e dos diferentes processos a que são submetidas, as fibras de madeira podem se apresentar com diferentes características físicas e químicas. Tais características podem influenciar as propriedades dos compósitos polímero/madeira produzidos (HILLIG et al., 2008).

É comum mencionar que são destinados para a geração de energia térmica nas indústrias do setor de base florestal e também em outros setores industriais, contudo amplos esforços devem ser feitos para abranger os múltiplos usos dos resíduos (SILVA, 2001 apud FONSECA, 2005)

\subsection{Processamento por extrusão em dupla-rosca}

Os processos de mistura e conformação dos compósitos poliméricos reforçados com fibras celulósicas podem ser os mais diversos, mas existe uma tendência, tanto na indústria quanto no desenvolvimento de trabalhos científicos, de se utilizar a extrusão dos materiais em extrusora de dupla-rosca, seguida da injeção da mistura para obtenção do produto final (SAHEB e JOG, 1999 apud HILLIG et al., 2008).

\section{MATERIAIS E MÉTODOS}

Os materiais que foram utilizados estão listados a seguir: O resíduo de pó de madeira, denominado (RPM), gentilmente cedido pela empresa Moveleiras Marisol Ltda, localizada em Campo Grande - RJ. O resíduo de madeira plástica (RMP), gentilmente cedido pela Companhia Municipal de Limpeza Urbana (COMLURB). As matérias primas utilizadas neste trabalho para a produção dos compósitos são provenientes de madeira e plásticos reciclados. Não foi empregado nenhum tipo de aditivo.

Para comparação foi utilizado polietileno de alta densidade virgem nas mesmas proporções de carga de pó de madeira, fornecido pela RIO POLÍMEROS S.A. Os equipamentos utilizados para a preparação e caracterização das misturas foram:

- Balança analítica MARTE AY2201

- Densímetro DSL910 - GEHAKA ${ }^{1}$

- Plastômetro MeltFlowQuik Index - CEAST'1 (pistão, matriz, pesos)

- Durômetro Shore D modelo GS7021

- Extrusora Dupla Rosca Marca TeckTrill, contra-rotatória, L/D 401

- Metalizador Baltec SCD $050^{2}$

- Equipamento para MEV -LEO $435 \mathrm{VP}^{2}$ 
Os equipamentos estão alocados nos seguintes locais:

Laboratório Didático de Polímeros - UEZO

Laboratório de Microscopia - UEZO

A Figura 1 mostra o diagrama de blocos com todas as etapas experimentais envolvidas neste trabalho. O desenvolvimento do trabalho foi dividido em quatro etapas.

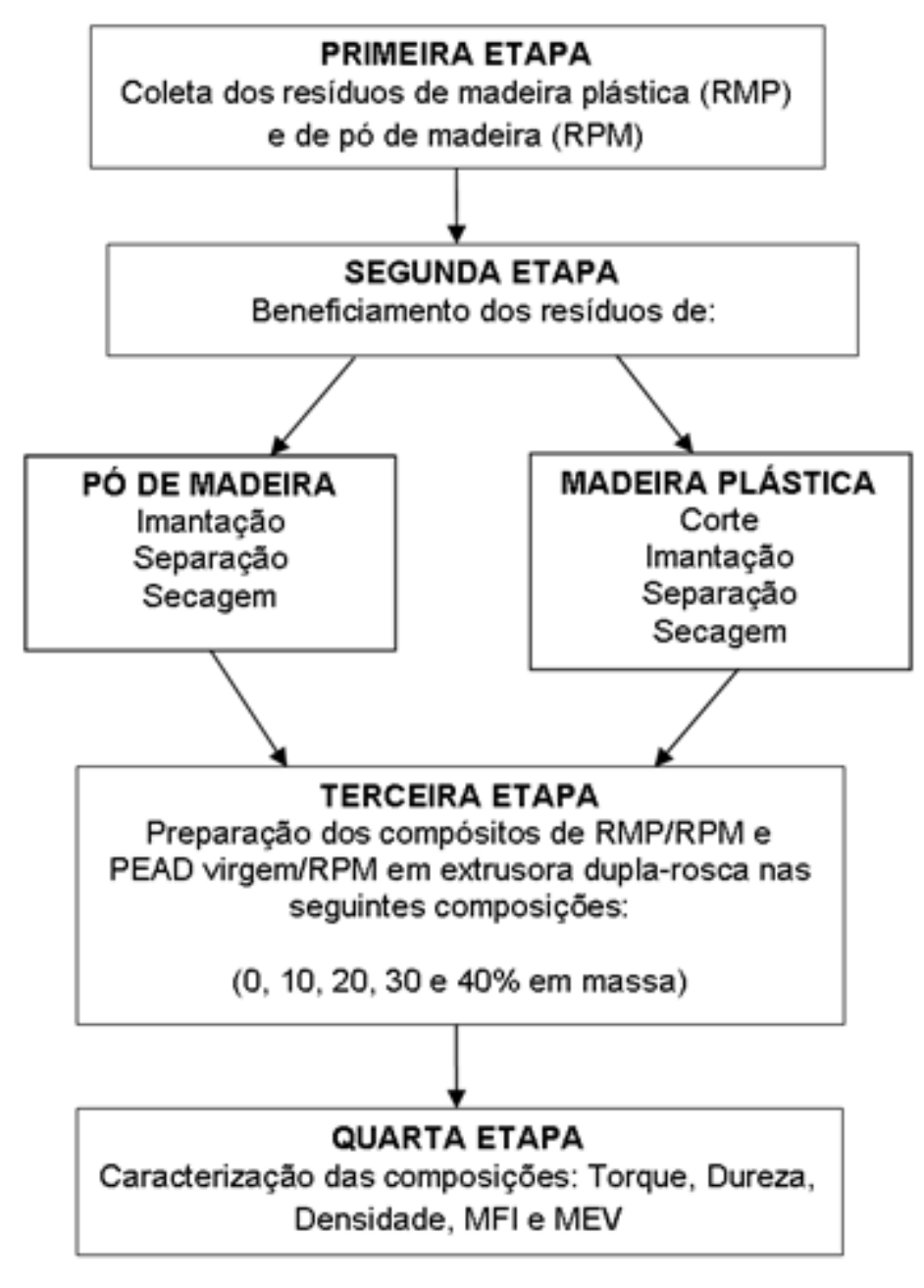

Figura 1: Fluxograma de trabalho

Fonte: Elaborado pelos autores (2015).

A Figura 2 mostra o RPM que foi cedido gentilmente pela empresa Moveleira Marisol Ltda. 


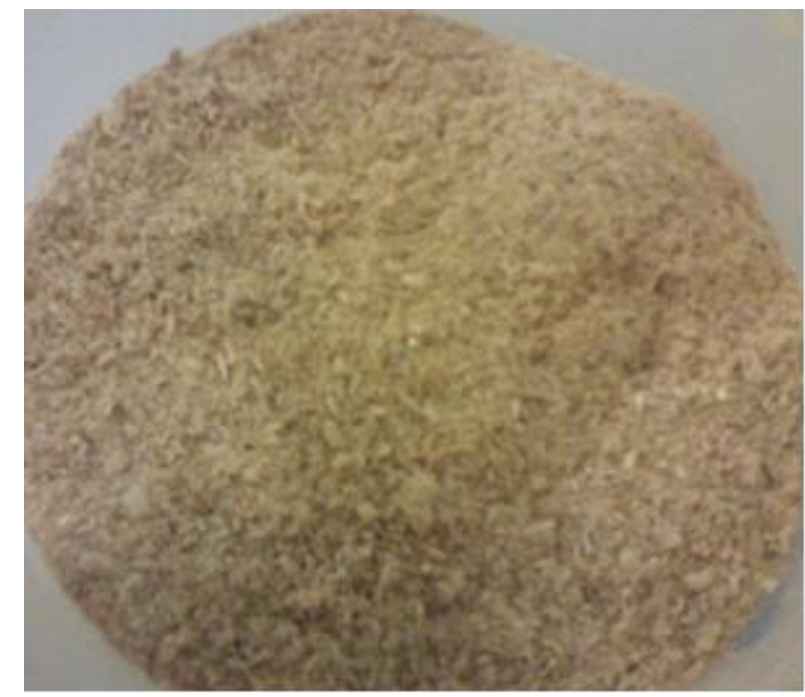

Figura 2: Resíduo de pó de madeira (RPM)

Fonte: Elaborado pelos autores (2015).

A figura 3 mostra o RMP que foi cedido gentilmente pela COMLURB.

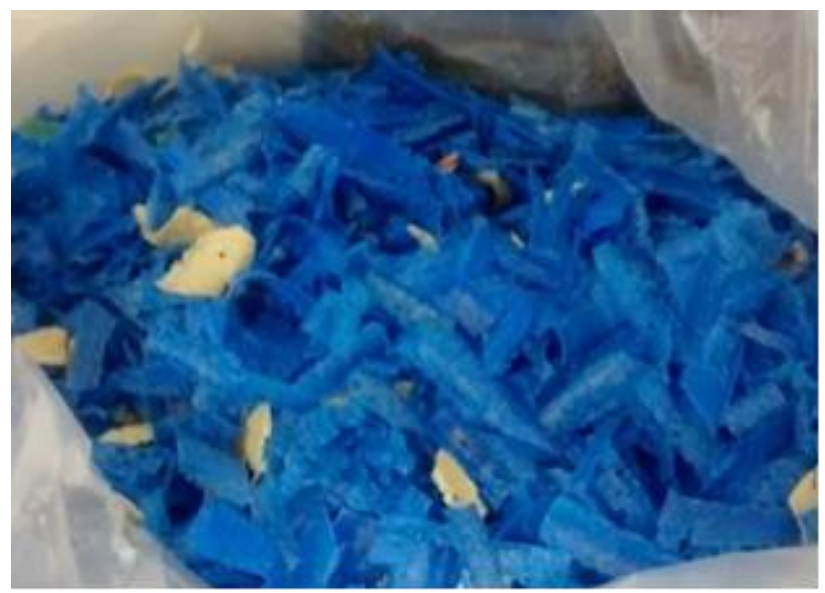

Figura 3: Resíduo de madeira plástica (RMP)

Fonte: Elaborado pelos autores (2015).

O beneficiamento do RMP foi realizado por meio de corte, imantação e separação física de outros resíduos presentes como madeira convencional de tamanho discrepante. O beneficiamento do RPM foi realizado por meio de imantação, separação e secagem.

Após o beneficiamento do RMP e RPM, os materiais foram pesados e misturados fisicamente de modo que cada proporção alcança-se $100 \mathrm{~g}$, com diferentes percentuais (em massa) de RPM (0, 10, 20, 30 e 40\%) utilizando uma velocidade de rosca de $60 \mathrm{rpm}$, e velocidade de alimentação para dosagem do material na extrusora de $6 \mathrm{rpm}$. Compósitos de PEAD-v e RPM foram processados para nível de comparação com o RMP, também beneficiados do mesmo modo e sem o uso de compatibilizantes.

As amostras de PEAD-v e RMP assim como os compósitos de PEAD-v e RMP com RPM foram processados em extrusora dupla rosca (TeckTrill contra-rotatória ${ }^{1}$ ), L/D 40, com 10 zonas de temperatura mais a zona de temperatura do cabeçote, utilizando uma velocidade de rosca de $60 \mathrm{rpm}$, e velocidade de alimentação para dosagem do material na extrusora de $6 \mathrm{rpm}$. O perfil de 
temperatura utilizado nas respectivas zonas de aquecimento foi de: $115^{\circ} \mathrm{C}, 135^{\circ} \mathrm{C}, 135^{\circ} \mathrm{C}, 150{ }^{\circ} \mathrm{C}$, $155^{\circ} \mathrm{C}, 160{ }^{\circ} \mathrm{C}, 170{ }^{\circ} \mathrm{C}, 170^{\circ} \mathrm{C}, 175^{\circ} \mathrm{C}, 175^{\circ} \mathrm{C}, 180{ }^{\circ} \mathrm{C}$ (cabeçote).

\section{RESULTADOS E DISCUSSÃO}

\subsection{Análise do torque}

A Figura 4 mostra os resultados de torque máximo apresentados pela extrusora durante o processamento dos compósitos. Misturas formadas pelo PEAD virgem e pelo resíduo de pó de madeira (pontos em preto). Misturas formadas pelo resíduo de madeira plástica e pelo resíduo de pó de madeira (pontos em vermelho), nos percentuais de pó de madeira de 0, 10, 20, 30 e 40\% em massa.

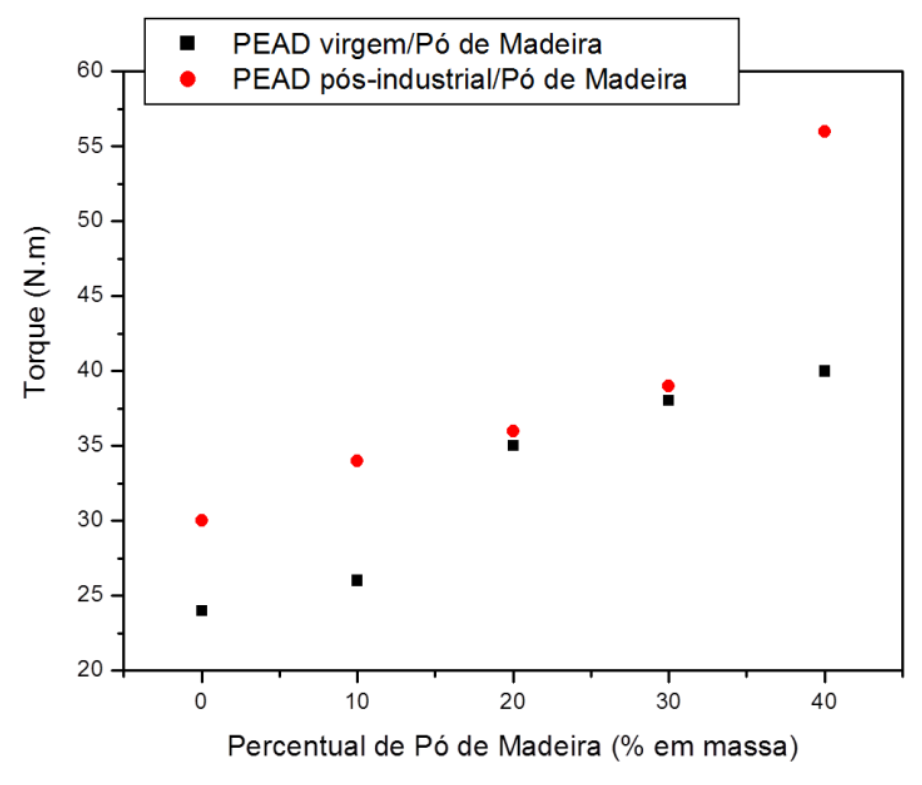

Figura 4: Resultados do torque dos compósitos extrusados Fonte: Elaborado pelos autores (2015).

Em todos os casos, observa-se que o torque aumentou com o aumento da carga nos sistemas, para todos os percentuais utilizados. Este fato já é esperado, uma vez que o torque está relacionado ao aumento de viscosidade e de dureza, quando se adiciona uma carga de reforço. Ainda, este aumento foi mais significativo para os compósitos com $10 \%$ e $40 \%$ em massa de pó de madeira, quando utilizado o resíduo de madeira plástica.

\subsection{Análise da dureza}

Utilizando a normativa a ASTM D 2240, a Figura 5 apresenta os resultados de dureza dos compósitos formados pelo PEAD virgem e pelo resíduo de pó de madeira (pontos em preto). Misturas formadas pelo resíduo de madeira plástica e pelo resíduo de pó de madeira (pontos em vermelho), nos percentuais de pó de madeira de 0,10, 20, 30 e 40\% em massa. 


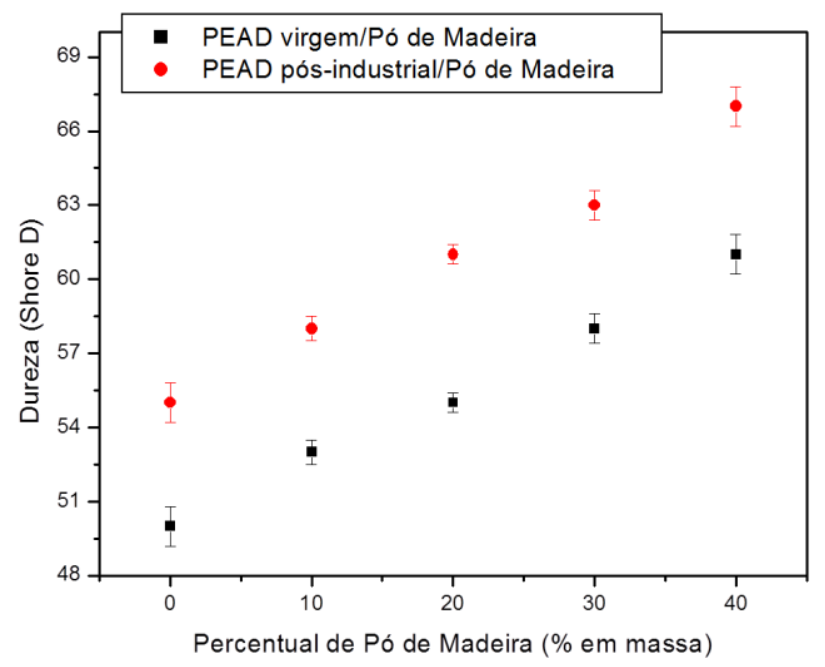

Figura 5: Resultados de dureza dos compósitos extrusados Fonte: Elaborado pelos autores (2015).

Observa-se um aumentou significativo de dureza em todas as proporções, sendo mais pronunciado para os compósitos formados com resíduos de madeira plástica com pó de madeira. Estes resultados sugerem que o pó de madeira esteja atuando como carga de reforço.

\subsection{Análise de densidade}

A Figura 6 apresenta os resultados das densidades dos compósitos formados pelo PEAD virgem e pelo resíduo de pó de madeira (pontos em preto) de acordo com a norma ASTM D7922008. Misturas formadas pelo resíduo de madeira plástica e pelo resíduo de pó de madeira (pontos em vermelho), nos percentuais de pó de madeira de 0, 10, 20, 30 e 40\% em massa.

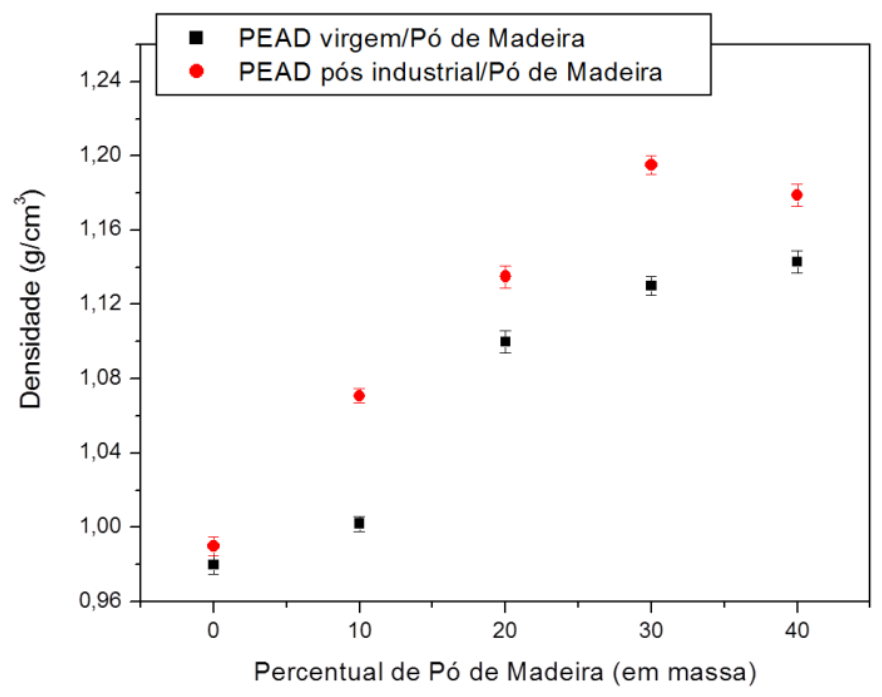

Figura 6: Resultados dos testes de densidade dos compósitos extrusados Fonte: Elaborado pelos autores (2015). 
Observa-se, em todos os compósitos formados, o aumento significativo da densidade, sendo mais pronunciado para os compósitos formados com resíduo de madeira plástica e de pó de madeira no percentual de $30 \%$ em massa de pó de madeira. Em $40 \%$ em massa, para o compósito com resíduo de madeira plástica, observa-se uma redução no valor de densidade, possivelmente devido a uma dificuldade de inserção na carga pelo aumento expressivo e visível da viscosidade do sistema, neste percentual de carga utilizado. Porém, este aumento expressivo na densidade para todos os compósitos sugerem que o resíduo de pó de madeira esteja atuando como carga de preenchimento.

\subsection{Análise do índice de fluidez}

A Figura 7 apresenta os resultados do índice de fluidez (MFI) dos compósitos formados pelo PEAD virgem e pelo resíduo de pó de madeira (pontos em preto), testes utilizando a ASTM D1238. Misturas formadas pelo resíduo de madeira plástica e pelo resíduo de pó de madeira (pontos em vermelho), nos percentuais de pó de madeira de 0,10, 20, 30 e 40\% em massa.

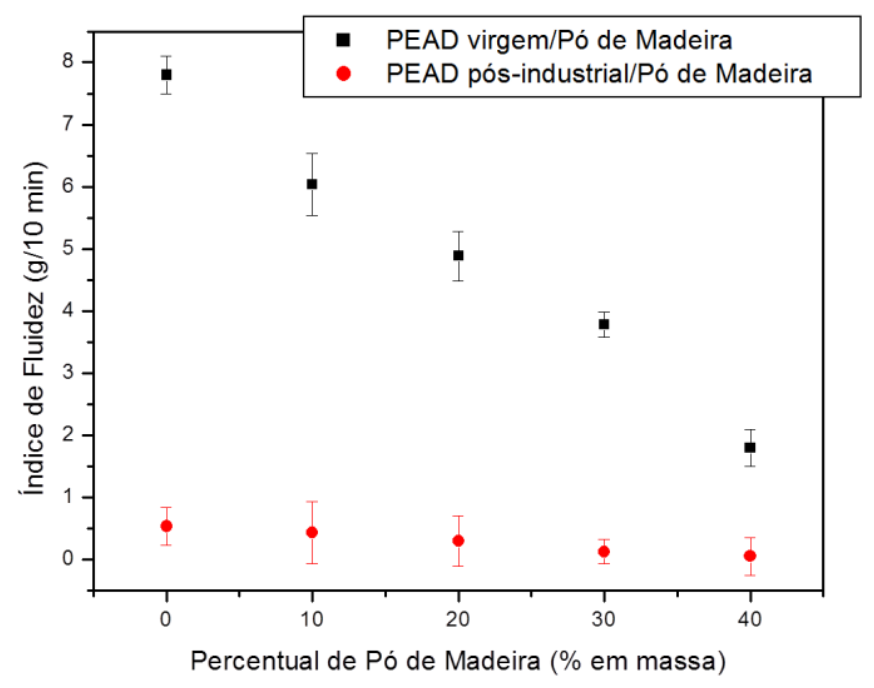

Figura 7: Resultados do Índice de Fluidez dos compósitos extrusados Fonte: Elaborado pelos autores (2015).

Pode-se observar que a variação entre o PEAD virgem e RMP gerou comportamentos distintos quanto ao índice de fluidez. Para os compósitos formados pelo PEAD virgem e pó de madeira, observa-se que o aumento no teor de pó de madeira promoveu uma drástica redução na fluidez, possivelmente devido à presença da carga estar influenciando significativamente a viscosidade do material, promovendo um aumento expressivo nesta, o que possivelmente ocasionou a redução na fluidez dos sistemas. Entretanto, este fato não é observado para os compósitos $100 \%$ reciclados. A fluidez praticamente não foi alterada pela presença da carga. Isto pode ter ocorrido devido a presença dos aditivos (pigmentos, auxiliares de processamento) presentes na madeira plástica, um resíduo pós-industrial. 


\subsection{Análise morfológica por microscopia eletrônica de varredura (MEV)}

As micrografias das superfícies de fratura dos compósitos formados pelo PEAD virgem e pelo resíduo de pó de madeira (Figura 8), nos percentuais de pó de madeira de 0, 10, 20, 30 e 40\% em massa (Figuras 8A, 8B, 8C e 8D, respectivamente).
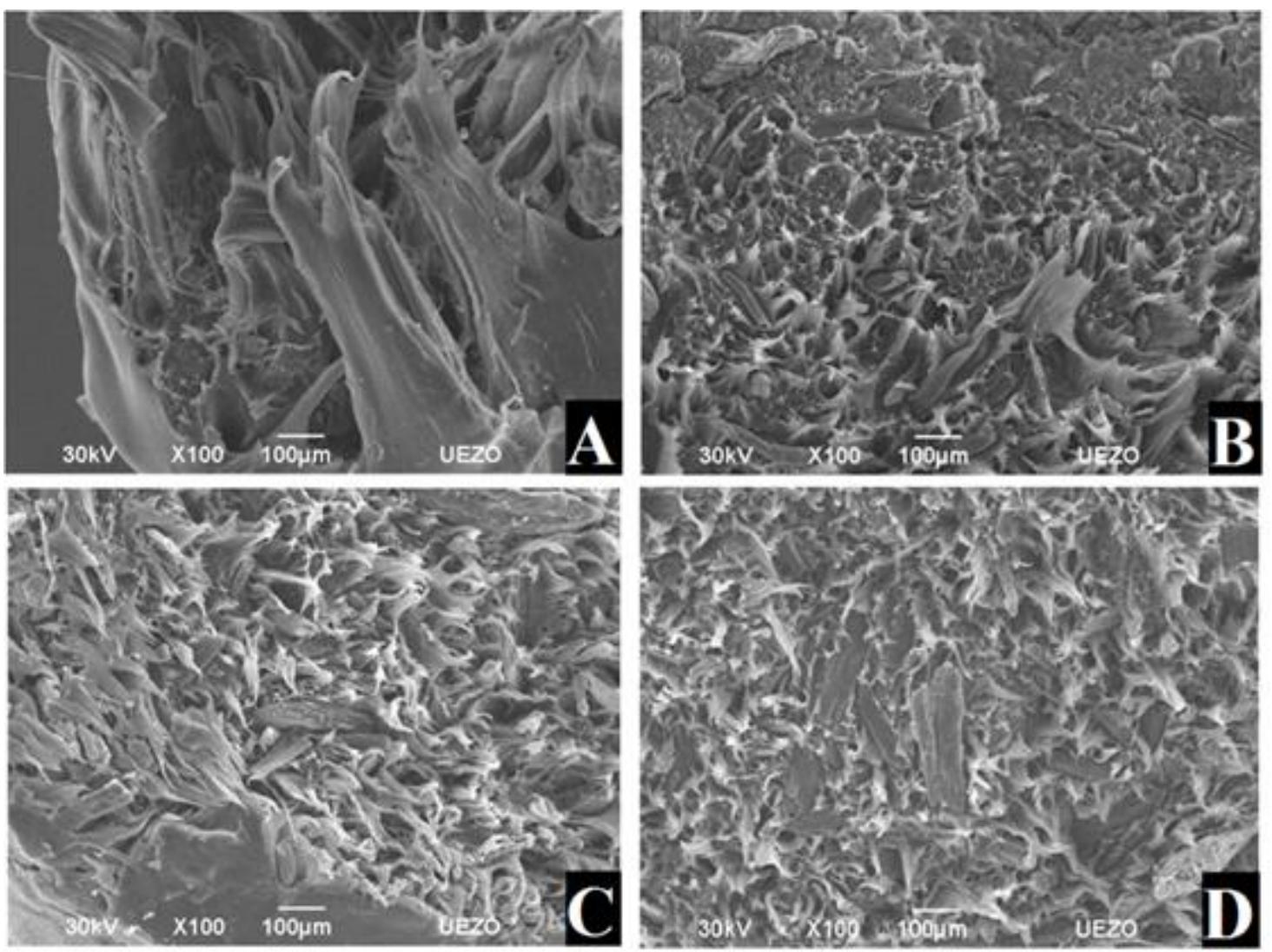

Figura 8: Compósitos de PEAD virgem com resíduo de pó de madeira nos percentuais: (A) 10\%; (B) $20 \%$; (C)30\% e (D) $40 \%$ em massa.

Fonte: Elaborado pelos autores (2015).

As imagens mostram que, em todos os casos, houve boa homogeneidade das misturas, sendo mais pronunciada nos compósitos com $30 \%$ e $40 \%$ em massa de resíduo de pó de madeira (figuras $13 \mathrm{C}$ e $13 \mathrm{D}$, respectivamente).

A figura 9 mostra as micrografias de MEV das superfícies de fratura dos compósitos formados pelo resíduo de madeira plástica e pelo resíduo de pó de madeira nos percentuais de pó de madeira de 0, 10, 20, 30 e 40\% em massa (Figuras 9A, 9B, 9C e 9D, respectivamente). 

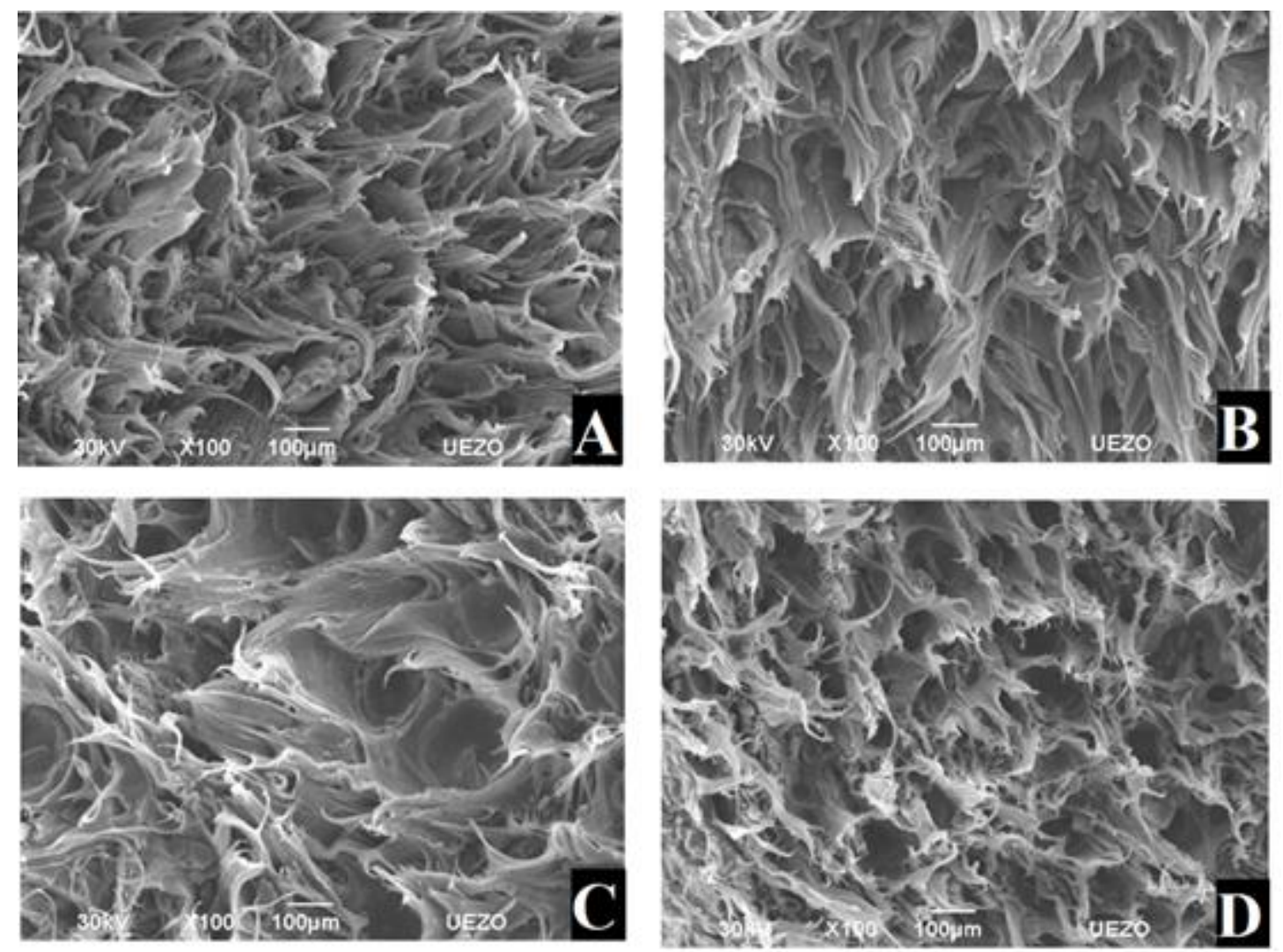

Figura 9: Compósitos de resíduo de madeira plástica com resíduo de pó de madeira nos percentuais: (A) $10 \%$; (B)20\%; (C)30\% e (D) $40 \%$ em massa

Fonte: Elaborado pelos autores (2015).

As imagens mostram que, em todos os casos, houve boa homogeneidade das misturas, não mostrando fase dispersa e matriz, separadamente, também sendo mais pronunciada nos compósitos com 30\% e 40\% em massa de resíduo de pó de madeira (figuras 8C e 8D, respectivamente).

\section{CONCLUSÃO}

Pode-se considerar que a madeira plástica é um produto inovador e com utilização diversificada e com viabilidade técnica comprovada. Porém, no Brasil existe certa relutância por parte das indústrias de transformação de termoplásticos em empregar o uso de farinha de madeira e outras cargas naturais devido à falta de familiaridade com esses resíduos. Outro fator é a diferença entre a indústria de transformação de plásticos e a de madeira, que gera algumas incompatibilidades, que vão desde a ausência de fornecedores em comum de matéria-prima e equipamentos a formas distintas de processamento de materiais em escalas diferentes.

A utilização de resíduo de pó de madeira em compósitos permite obter ganhos significativos nas propriedades físicas em comparação ao polímero puro. Dentre as propriedades estudadas, a que mais sofreu influência da adição de madeira foi a densidade na proporção de 70/30 para RMP/RPM e o torque na proporção de 60/40 para RPM/RMP. A densidade manteve uma diferença regular em todas as proporções, sendo um pouco mais perceptível em 80/20 para RMP/ RPM.

Os resultados indicaram que o resíduo de pó de madeira tende a aumentar a viscosidade do compósito com crescimento linear, assim como os resultados com os ensaios de dureza mostraram 
um aumento constante em todas as amostras. A densidade também foi aumentada de modo considerável em todas as proporções. De acordo com os resultados de densidade e de dureza, conclui-se que o pó de madeira atuou como carga reforço e carga de preenchimento.

Deste modo, os resultados mostraram que é perfeitamente viável a produção de compósitos com resíduos de madeira plástica e pó de madeira com melhoria de propriedades. Com isto, pode-se concluir que o presente trabalho contribui para o meio ambiente, uma vez que promove a produção de materiais ecologicamente corretos.

Como sugestões para trabalhos, destacam-se: Finalizar as análises de Termogravimetria (TGA) e Calorimetria Diferencial de Varredura (DSC) para avaliar a análise térmica dos compósitos formados; Realizar ensaios mecânicos de tração, flexão e resistência ao impacto dos materiais, para verificar o comportamento mecânico dos materiais formados; Realizar o ensaio balístico para verificar se os compósitos podem ser aplicados nesta área.

\section{AGRADECIMENTOS}

Agradeço primeiramente à Deus que me permite ter saúde para aprender sempre mais. À minha família e esposa que me apóia e ajuda nas minhas conquistas. Agradecimento em especial a minha orientadora que sempre me apoiou nos desafios acadêmicos.

\section{REFERENCIAS}

CORREA, C.A. et al - Compósitos termoplásticos com madeira. Polímeros: Ciência e Tecnologia, vol.13, nº3, p. 154$165,2003$.

FONSECA, F. M. C., Desenvolvimento e caracterização de compósitos à base de polietileno de alta densidade (PEAD) reciclado e fibras vegetais. Dissertação de Mestrado Rede Temática de Engenharia de Materiais Universidade Federal de Ouro Preto. Belo Horizonte, 2005.

GUAMÁ, F. F. M. C. et al. Lixo Plástico - de sua produção até a madeira plástica. XXVIII Encontro Nacional de Engenharia de Produção. Anais..., RJ, Brasil, 13 a 16 de outubro de 2008.

HILLIG, E. et al. - Caracterização de compósitos produzidos com polietileno de alta densidade (HDPE) e serragem da indústria moveleira. Revista Árvore, Viçosa-MG, v.32, n.2, p.299-310, 2008.

MANO, E. B. Introdução a polímeros. São Paulo: Edgard Blucher. 2004.

OLANDOSKI, D.P. Rendimento, resíduos e considerações sobre melhorias no processo em indústrias de chapas compensadas. 2001 85f. Dissertação (Mestrado em Ciências Florestais). Universidade Federal do Paraná, Curitiba, 2001 . 